\title{
Modelos Assistenciais na Enfermagem - Revisão de Literatura
}

Recebido em: 24/04/2012

Aceito em: 30/01/2013

Fátima Silvana Furtado Gerolin ${ }^{1}$ Isabel Cristina Kowal Olm Cunha ${ }^{2}$

Com o objetivo de realizar uma revisão da literatura norteada pelas questões: quais as contribuições de trabalhos disponíveis on-line na base de dados PUBMED, SciELO e Web of Science com temas relacionados a modelos assistenciais na área de enfermagem, no recorte temporal de 2004 a 2011, e quais os modelos assistenciais encontrados com maior frequência, foram selecionados 60 artigos diretamente vinculados ao objeto da pesquisa. Foram classificados segundo país de origem, nomenclatura do modelo e características dos dois modelos com mais publicações. Dos 11 modelos identificados, predominam: Prática Baseada em Evidência e Modelo Assistencial Centrado no Paciente.

Descritores: Assistência de Enfermagem, Assistência Centrada no Paciente, Enfermagem, Prática Clínica Baseada em Evidências.

\section{Nursing Care Models - Literature Review}

With the purpose of carrying out a literature review guided by the following questions: What are the inputs of works available online in the database of PUBMED, Scielo, and Web of Science in the themes related to nursing care models, from 2004 to 2011, and what are the care models most frequently found? 60 articles directly related to the subject of the research were selected. They were sorted by country of origin, nomenclature of the model, and characteristics of the two models with the highest number of publications. From the 11 models identified, the prevailing ones are: Evidence-Based Practice and Patient-Oriented Care Model

Descriptors: Nursing Care, Patient-Oriented Care, Nursing, Evidence-Based Clinical Practice.

\section{Modelos Asistenciales en la Enfermería - Revisión de Literatura}

Con el objetivo de realizar una revisión de la literatura norteada por las cuestiones: cuáles son las contribuciones de trabajos disponibles online en la base de datos PUBMED, Scielo y Web of Science con temas relacionados a modelos asistenciales en el área de enfermería, en el recorte temporal del 2004 al 2011, y cuáles son los modelos asistenciales encontrados con mayor frecuencia, se seleccionaron 60 artículos directamente vinculados al objeto de la investigación. Se clasificaron según el país de origen, nomenclatura del modelo y características de los dos modelos con más publicaciones. De los 11 modelos identificados, predominan: Práctica Basada en Evidencia y Modelo Asistencial Centralizado en el Paciente.

Descriptores: Asistencia de Enfermería, Asistencia Centralizada en el Paciente, Enfermería, Práctica Clínica Basada en Evidencias.

\section{INTRODUÇÃO}

M uito se tem discutido sobre alterações ocorridas na gestão hospitalar em decorrência de mudanças sociopolítico-econômicas, trazendo impacto em aspectos administrativos e assistenciais. Para que os hospitais se mantenham sustentáveis e competitivos, os profissionais que fazem parte de seu corpo funcional vivenciam dificuldades cada vez maiores para gerenciar com qualidade, buscando resultados competitivos. Por consequência, os enfermeiros que tradicionalmente são responsáveis pela gerência do cuidado, tiveram que, ao longo de sua história, se adaptar e se preparar para novos desafios, ampliando sua área de atuação e de conhecimento. Essa busca fez com que ele compreendesse questões estratégicas do negócio, o que, no passado, não era discutido como sendo uma competência essencial para esse profissional.

Conhecimentoorganizacionalédefinidocomo:"Conhecimento é uma mistura fluida de experiências condensadas, valores, informação contextual e insight experimental, a qual proporciona uma estrutura para a avaliação e incorporação de novas experiências e informações". Ele tem origem e é aplicado na mente dos conhecedores. Nas organizações, ele costuma estar embutido não só em documentos ou repositórios, mas também em rotinas, processos, práticas e normas organizacionais ${ }^{(1: p .6)}$.

O processo de trabalho da Enfermagem particulariza-se em uma rede ou subprocessos que são denominados cuidar ou assistir, administrar ou gerenciar, pesquisar e ensinar. Cada um desses pode ser tomado como um processo à parte com seus próprios elementos (objeto, meios/instrumentos e atividade) que podem ou não coexistir em determinado momento e instituição. Nesses diferentes processos, os agentes, ou seja, os trabalhadores de enfermagem, inserem-se de forma heterogênica e hierarquizada, expressando a divisão técnica e social do trabalho(2). Observamos uma divisão de trabalho de enfermagem, segundo a qual cabe à categoria de nível médio, majoritariamente, as atividades assistenciais,

1 Enfermeira. Mestre em Enfermagem. Superintendente Assistencial do Hospital Alemão Oswaldo Cruz. Doutoranda da Escola Paulista de Enfermagem e membro do Grupo de Estudos e Pesquisa em Administração em Saúde e Gerencimento de Enfermagem - GEPAG, da Universidade Federal de São Paulo - UNIFESP. E-mail: fsilvanaf@gmail.com 2 Enfermeira. Professora Livre-Docente Chefe do Departamento de Administração e Saúde Coletiva e líder do GEPAG da EPE/ UNIFESP. 
e ao enfermeiro as ações de gerenciamento do cuidado e da unidade. Especificamente sobre o trabalho do enfermeiro, evidencia-se que as intervenções desse agente concretizam dois diferentes processos de trabalho: o processo "cuidar" e o processo "administrar", com a primazia do último ${ }^{(3)}$.

Uma pesquisa bibliográfica sobre as especificidades dos elementos que compõem os processos de trabalho de enfermagem resultou em duas frentes sobre o objeto de trabalho do enfermeiro: o cuidado e o gerenciamento desse cuidado. Ambos os objetos expressam a dupla dimensão intrínseca ao processo de trabalho do enfermeiro - o trabalho assistencial e o gerencial ${ }^{(4)}$.

Existe, portanto, um constante movimento do repensar a gestão e a assistência de enfermagem, sempre em busca de melhores práticas baseadas em evidências, que otimizem recursos e garantam uma assistência segura, organizada, com foco no cliente, pautada nas relações interpessoais que envolvem a Enfermagem, o paciente e a família. Nossas economias e sociedades mudaram substancialmente ao longo das últimas décadas, mas os métodos e as práticas de gestão continuam as mesmas. Práticas que por muito tempo consideramos padrão, hoje devem ser questionadas ${ }^{(5)}$.

Modelo de gestão constitui-se em uma configuração conceitual e operacional com características para estruturar os elementos do processo de trabalho e da organização dos recursos materiais, humanos e financeiros, definidos para uma dada estrutura institucional. Refere-se ao processo ativo de determinação e orientação a ser seguido para a realização dos objetivos institucionais, compreendendo um conjunto de análises, decisões, comunicação, liderança, motivação e controle/avaliação(6).

Com o fortalecimento das políticas públicas, a gestão na área da saúde passou a ter um novo olhar. A Estratégia da Saúde da Família - ESF valorizou a gestão por indicadores, avaliação dos serviços de saúde, processos de acreditação na área da atenção primária, bem como a busca por melhores resultados, associando ações na gestão, na prática do dia-a-dia, trazendo resultados mensuráveis. Isso motivou também a formação dos profissionais na área da saúde, no sentido de trazer conteúdos relacionados às práticas de gestão, possibilitando aos profissionais um repensar da sua atuação, não apenas no fazer, mas no gerir para resultados produtivos.

Nesse sentido, se o modelo de gestão tem sido questionado, o momento também é oportuno para reflexões sobre o modelo assistencial. O modelo assistencial diz respeito ao modo como são organizadas, em uma dada sociedade, as ações de atenção à saúde, envolvendo os aspectos tecnológicos e assistenciais. Ou seja, é uma forma de organização e articulação entre os diversos recursos físicos, tecnológicos e humanos disponíveis para enfrentar e resolver os problemas de saúde de uma coletividade ${ }^{(7)}$.

\section{MODELOS ASSISTENCIAIS}

Em referenciais teóricos que abordam essa busca por modelos assistenciais aplicáveis à realidade brasileira, destaca-se a publicação ${ }^{(8)}$ que constata a evolução da prática de enfermagem marcada por uma falta de profissionais qualificados de nível superior para atender à demanda. Dessa forma, tornam-se necessárias adaptações dos modelos importados de países onde o enfermeiro representa a força de trabalho principal na equipe de enfermagem.

A discussão relacionada ao modelo assistencial, muitas vezes pouco compreendido na sua totalidade, traz a reflexão de como se pode definir a assistência hospitalar no Brasil face às diferenças econômicas, sociais e políticas quando comparada com outros países e mesmo entre as diversas regiões brasileiras.

Observa-se que a Enfermagem brasileira tem buscado, ao longo de sua história, modelos de gestão na assistência para utilização principalmente na área hospitalar. Esses modelos, muitas vezes desenvolvidos e aplicados em outros países, passam por adequações na tentativa de aplicação para a realidade brasileira.

\section{OBJETIVO}

Este artigo teve por objetivo realizar uma revisão sistemática da literatura norteada pelas seguintes questões:

1. Quais as contribuições de trabalhos disponíveis on-line em uma base de dados com temas relacionados a modelos assistenciais na área de enfermagem?

2. Quais os modelos assistenciais encontrados com maior frequência?

\section{METODOLOGIA}

Estudo de revisão sistemática através da análise de artigos científicos disponíveis on line nas bases de dados PUBMED, SciELO e Web of Science no recorte temporal de 2004 a 2011, identificados através das palavras-chaves"Models, Nursing". A estratégia de busca utilizada foi ("models, nursing"[MeSH Terms] OR ("models"[All Fields] AND "nursing"[All Fields]) OR "nursing models"[All Fields] OR ("nursing"[All Fields] AND "models"[All Fields])) AND ("humans"[MeSH Terms] AND English[lang] AND ("2004/01/01"[PDAT] : "2011/12/31"[PDAT])).

Dos 1.240 artigos localizados, selecionaram-se 60 por estarem diretamente vinculados ao objeto da pesquisa, através da leitura dos títulos e resumos dos mesmos. Foram classificados segundo país de origem e nomenclatura do modelo assistencial. Elaborou-se, então, uma tabela com sumário das características dos estudos relacionados aos modelos assistenciais: Modelo de Prática Baseado em Evidência e Modelo Assistencial Centrado no Paciente.

\section{RESULTADOS E DISCUSSÃO}

Identificou-se que $14(23,3 \%)$, do total dos 60 artigos encontrados, são revisões teóricas e oito (13,3\%) foram testados em instituições de saúde e/ou atendimento domiciliar. Em relação ao país onde foi desenvolvida a experiência relatada, 41 $(68,3 \%)$ ocorreram nos Estados Unidos, nove (15\%) na Inglaterra, três (5\%) na Austrália, três (5\%) no Canadá, dois (3,3\%) na Nova 
Zelândia, um(1,6\%) na África do Sul e um (1,6\%) na Espanha.

Analisando os 60 artigos, identificaram-se 11 diferentes modelos assistenciais com nomenclatura definida e respectivas quantidades de artigos que descrevem o modelo, que podem ser visualizados na Tabela 1 abaixo.

Tabela 1 - Distribuição das publicações segundo nomenclatura do Modelo Assistencial identificado. São Paulo, 2012

\begin{tabular}{|l|l|l|}
\hline Nomenclatura & $N^{\circ}$ & $\%$ \\
\hline Modelo de Prática Baseada em Evidência & 10 & 37,03 \\
Modelo Assistencial Centrado no Paciente & 08 & 29,62 \\
Sistema Clínico Avançado & 01 & 3,7 \\
Modelo Baseado no Significado Social & 01 & 3,7 \\
Primary Nursing & 01 & 3,7 \\
Cuidado Restaurativo para Autonomia Assistida & 01 & 3,7 \\
\hline Cuidado Holístico & 01 & 3,7 \\
\hline Modelo de Sistema Neumann & 01 & 3,7 \\
Modelo Colaborativo de Prestação de Cuidado & 01 & 3,7 \\
Cuidado Baseado na Espiritualidade & 01 & 3,7 \\
Modelo de Cuidado Whanaungatanga & 01 & 3,7 \\
\hline Total & 27 & 100 \\
\hline
\end{tabular}

Dos 11 modelos identificados, a predominância é a do Modelo de Prática Baseada em Evidência, seguido do Modelo Assistencial Centrado no Paciente. Esses permitem a análise e a reflexão em relação aos modelos descritos, contribuindo para que os enfermeiros gestores repensem o modelo assistencial instituído nos hospitais face à realidade econômica, social e política.

Os artigos que tratam do Modelo de Prática Baseada em Evidência ${ }^{(9-18)}$, enfatizam o fato de que o enfermeiro é o centro do processo, pois é ele quem observa, avalia, questiona, comunica e implementa novos conhecimentos na prática clínica.

Os artigos que se referem ao Modelo de Cuidado Centrado no Paciente ${ }^{(19-26)}$ consideram que deve ser levado em consideração o ponto de vista do paciente. A função básica desse modelo é tornar o paciente informado e envolvido com a tomada de decisão em relação aos seus cuidados, fazendo-o sentir-se apoiado, confortável e confiante. Os principais objetivos do Cuidado Centrado no Paciente são reduzir danos decorrentes da assistência e reduzir o sofrimento.

É consenso entre esses dois modelos que os profissionais precisam ter bom conhecimento da prática clínica, habilidade técnica e comunicação.

Dos 18 artigos que estão relacionados aos dois modelos mais encontrados - "Modelo de Prática Baseado em Evidência" e "Modelo Assistencial Centrado no Paciente"-, 12 $(66,6 \%)$ são relatos de experiência, cinco $(27,7 \%)$ são artigos de reflexão e um (5,5\%), revisão sistemática.

Nos artigos analisados, buscou-se ainda verificar em que revistas foram publicados, informação disponibilizada na Tabela 2, a seguir.
Tabela 2 - Distribuição das publicações segundo nome da revista. São Paulo, 2012.

\begin{tabular}{|c|c|}
\hline Nome da Revista & Artigos* \\
\hline Journal of Nursing Administration & 8 \\
\hline Nursing Science Quarterly & 7 \\
\hline The Nursing Clinics of North America & 5 \\
\hline Journal of Nursing Management & 4 \\
\hline Journal of the American Academy of Nurse Practitioners & 2 \\
\hline Nursing Outlook & 2 \\
\hline Nursing Praxis in New Zealand inc & 2 \\
\hline Nursing Standard (Royal College of Nursing - Great Britain) & 2 \\
\hline British Journal of Nursing & 2 \\
\hline Alberta Association of Registered Nurses & 1 \\
\hline The Queensland Nurse & 1 \\
\hline $\begin{array}{l}\text { Contemporary Nurse : a journal for the Australian } \\
\text { nursing profession }\end{array}$ & 1 \\
\hline $\begin{array}{l}\text { Journal of PeriAnesthesia Nursing : officia journal } \\
\text { of the American Society of PeriAnesthesia Nurses / } \\
\text { American Society of PeriAnesthesia Nurses }\end{array}$ & 1 \\
\hline Nursing Administration Quarterly & 1 \\
\hline Clinical Nurse Specialist - CNS & 1 \\
\hline Texas Nursing & 1 \\
\hline The American Journal of Nursing & 1 \\
\hline International Nursing Review & 1 \\
\hline Journal of Nursing Care Quality & 1 \\
\hline Geriatric Nursing & 1 \\
\hline Journal of Family Nursing & 1 \\
\hline $\begin{array}{l}\text { Joint Commission Journal on Quality and Patient } \\
\text { Safety / Joint Commission Resources }\end{array}$ & 1 \\
\hline Dimensions of Critical Care Nursing & 1 \\
\hline $\begin{array}{l}\text { Medsurg Nursing : official journal of the Academy } \\
\text { of Medical-Surgical Nurses }\end{array}$ & 1 \\
\hline Journal of Continuing Education in Nursing & 1 \\
\hline Curationis & 1 \\
\hline International Journal of Nursing Practice & 1 \\
\hline The Journal of Nursing Education & 1 \\
\hline $\begin{array}{l}\text { Worldviews on Evidence-Based Nursing / Sigma } \\
\text { Theta Tau International, Honor Society of Nursing }\end{array}$ & 1 \\
\hline Nursing Leadership (Toronto, Ont.) & 1 \\
\hline Journal of Clinical Nursing & 1 \\
\hline $\begin{array}{l}\text { Journal of Christian Nursing : a quarterly } \\
\text { publication of Nurses Christian }\end{array}$ & 1 \\
\hline Critical Care Nursing Quarterly & 1 \\
\hline Nursing Outlook & 1 \\
\hline TOTAL 33 Revistas & 59 artigos \\
\hline
\end{tabular}

* artigos localizados por revista

Observamos que as quatro revistas que mais apresentaram publicações relacionadas a modelos assistenciais foram: Journal of Nursing Administration (08 $=24 \%)$, Nursing Science Quarterly $(07=21 \%)$, The Nursing Clinics of North America (05 $=15 \%$ ) e Journal of Nursing Management $(04=12 \%)$. 
Neste estudo, no recorte temporal proposto de 2004 a 2011, os artigos localizados na base de dados PUBMED nos permitem afirmar que tanto instituições de saúde como de ensino de enfermagem vêm desenvolvendo estudos e pesquisas objetivando testar modelos assistenciais e, assim, buscando melhor qualificar a assistência prestada. E os modelos assistenciais mais publicados são: Modelo Assistencial Baseado em Evidência e o Modelo Centrado no Paciente. Apesar de diferentes na descrição dos processos para sua construção e implantação, estes levam em consideração o paciente, respeitando seus valores e preferências, e têm como objetivo a segurança na assistencial. Os artigos discorrem sobre a origem dos modelos, como implantá-los, a relação com busca da qualidade na assistência prestada ao paciente, porém, sem evidências que comprovem que o modelo seja eficaz para aquela instituição.

\section{CONSIDERAÇÕES FINAIS}

Não importa o tipo de instituição de saúde, seja ela pública, privada, relacionada ou não a alguma instituição de ensino, hospital geral, hospital especializado, entre outros, faz-se necessário entender a realidade de cada estrutura para daí escolher ou desenvolver um modelo assistencial possível de ser aplicado e que traga resultados satisfatórios.

Diante deste levantamento, observamos que dentre os estudos publicados não se encontram publicações brasileiras, o que pode estar relacionado à base de dados consultada. Não foram encontrados artigos que comparam o modelo assistencial frente ao modelo de gestão da instituição de saúde envolvida, sendo necessário um estudo mais aprofundado, cruzando esses dois temas.

\section{Referências}

1. Davenport TH, Prusak L. Conhecimento empresarial: como as organizações gerenciam seu conhecimento intelectual. $3^{\mathrm{a}}$ ed. Rio de Janeiro: Campus;

2001. p. 6.

2. Silva VEF. O desgaste do trabalhador de enfermagem: a relação trabalho de enfermagem e saúde do trabalhador [tese]. São Paulo: Escola de Enfermagem da USP; 1996.

3. Felli VEA, Peduzzi M. O trabalho gerencial em enfermagem. In: Kurcgant P. Gerenciamento em enfermagem. Rio de Janeiro: Guanabara Koogan; 2005.

4. Peduzzi M, Anselmi ML. O processo de trabalho de enfermagem: a cisão entre planejamento e execução do cuidado. Rev Bras Enferm. 2002;55(4):392-8.

5. Pflaeging N. Liderando com metas flexíveis. Porto Alegre: Bookman; 2009.

6. Souza AS. A construção de um modelo de gestão para o CA/HU/UFJF: as rotas de sua operacionalidade. Rev Faculd Serviço Soc Libertas. 2004/2005;4/5(n esp):1-16.

7. Morosini MVGC, Corbo ADA, orgs. Modelos de atenção e a saúde da família [Internet]. [citado em 2011 Mar 26]. Disponível em: http://www.epsjv.fiocruz.br/ index.php?Area=Material $\& \mathrm{MNU}=\& T i p o=1 \& \mathrm{Num}=26$

8. Magalhães AMM, Juchem BC. Primary nursing: adaptando um novo modelo de trabalho no serviço de enfermagem cirúrgica do Hospital de Clínicas de Porto Alegre. Rev Gaúcha Enferm. 2000;21:5-18.

9. Olade RA. Strategic collaborative model for evidence-based nursing practice. Worldviews Evid Based Nurs. 2004;1 (1):60-8.

10. Balakas K, Potter P, Pratt E, Rea G, Williams J. Evidence equals excellence: the application of an evidence-based practice model in an academic medicalcenter. Nurs Clin North Am. 2009;44(1):1-10.

11. Reavy $K$, Tavernier $S$. Nurses reclaiming ownership of their practice: implementation of an evidence-based practice model and process. J Contin Educ Nurs. 2008;39(4):166-72.

12. Strout TD, Lancaster K, Schultz AA. Development and implementation of an inductive model for evidence-based practice: a grassroots approach for building evidence-based practice capacity in staff nurses. Nurs Clin North Am. 2009;44(1):93-102.
13. Newhouse RP, Dearholt S, Poe S, Pugh LC, White KM. Organizational change strategies for evidence-based practice. J Nurs Adm. 2007;37(12):552-7.

14. Levin RF, Keefer JM, Marren J, Vetter M, Lauder B, Sobolewski S. Evidence-based practice improvement: merging 2 paradigms. J Nurs Care Qual. 2010;25(2):117-26. 15. Waddell AW. Cultivating quality: shared governance supports evidence-based practice. Am J Nurs. 2009;109(11):53-7.

16. Honess C, Gallant P, Keane K. The Clinical Scholar Model: evidence-based practice at the bedside. Nurs Clin North Am. 2009;44(1):117-30.

17. Cullen L, Adams S. An evidence-based practice model. J Perianesth Nurs. 2010;25(5):307-10.

18. Abad-Corpa E, Meseguer LC. ET al Effectiveness of the implementation of an evidence-based nursing model using participatory action research in oncohematology: research protocol. J Adv Nurs. 2010;66:5-5.

19. E-Morris M, Caldwell B, Mencher KJ, Grogan K, Judge-Gorny M, Patterson Z, et al. Nurse-directed care model in a psychiatric hospital: a model for clinical accountability. Clin Nurse Spec. 2010;24(3):154-60.

20. Jasovsky DA, Morrow MR, Clementi PS, Hindle PA. Theories in action and how nursing practice changed. Nurs Sci Q. 2010;23(1):29-38.

21. Mitchell GJ, Bournes DA, Hollett J. Human becoming-guided patient-centered care: A new model transforms nursing practice. Nurs Sci Q. 2006;19(3):218-24.

22. Mitchell PH. Patient-centered care - a new focus on a time-honored concept. Nurs Outlook. 2008;56(5):197-8.

23. Pelzang R. Time to learn: understanding patient-centred care. Br J Nurs. 2010;19(14):912-7.

24. Hobbs JL. A dimensional analysis of patient-centered care. Nurs Res. 2009;58(1):52-62.

25. Wolf D, Lehman L, Quinlin R, Rosenzweig M, Friede S, Zullo T, et al. Can nurses impact patient outcomes using a patient-centered care model? J Nurs Adm. 2008;38(12):532-40.

26. Kelleher S. Providing patient-centred care in an intensive care unit. Nurs Stand. 2006;21(13):35-40. 